\title{
Durchfall bei Kindern und Jugendlichen - Tipps für die Praxis
}

\author{
Gespräch mit Prim. MedR. Ass.-Prof. DDr. Peter Voitl, MBA, \\ Kindergesundheitszentrum Donaustadt, Wien.
}

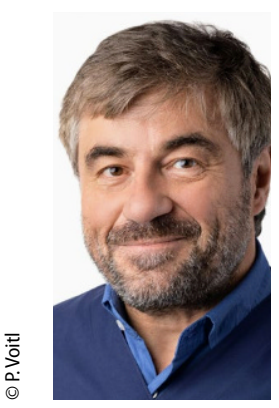

Prim. DDr. Peter Voitl
Pädiatrie \& Pädologie: In Zeiten der SARS-CoV-2 Pandemie kommen bestimmt weniger Patienten zu lhnen ins Zentrum. Bieten Sie webbasierte u/o telefonische Sprechstunden an? Voitl: Selbstverständlich bieten wir die medizinische Beratungen und Sprechstunden an. Das hat aber natürlich Grenzen, neue Patienten oder Patienten mit akut aufgetretenen Erkrankungen können telemedizinisch nicht seriös betreut werden. Andere Patienten, die wir seit langem kennen und bei denen eine Kontrolle ausständig ist, können aber sehr gut telefonisch beraten werden.

Kontaktieren Eltern Sie häufig wegen Durchfalls ihrer Kinder?

Ja. Durchfall ist ein sehr häufiges Phänomen vor allem Kleinkindesalter und somit oft Grund einer Konsultation unserer Ordination.

Sind eher kleine und ältere Kinder von Durchfall betroffen, oder auch Jugendliche?

Infektiöse Durchfallserkrankungen sind vor allem bei kleinen Kindern und Kindergartenkindern sehr häufig, weil der Kontakt im Kindergarten eng ist und auch bei bester Hygiene ein Austausch von Krankheitserregern stattfinden kann. Bei Jugendlichen kommt es auch zu Durchfallserkrankungen, hier ist aber auch eine andere Ursache, wie eine entzündliche Darmkrankheit, auszuschließen.

Gibt es saisonale Häufungen des Durchfalls in Österreich, und wann?

Das Interview führte Dr. Renate LesskyHöhl, 26.04.2020 fallserkrankungen, so tritt etwa die Campylobacter-Enteritis gehäuft in der warmen Jahreszeit (Juni bis September) auf. Die Rotavirusinfektion tritt gehäuft in den Monaten Februar bis April auf. Generell kann man aber von einer Häufung in der kalten Jahreszeit ausgehen.

Separieren Sie Kinder und Jugendliche, die an Durchfall leiden, im Wartezimmer Ihres Zentrums von den anderen wartenden Kindern und Jugendlichen?

Ja. Wir bieten eigene Ordinationszeiten für potentiell infektiöse $\mathrm{Pa}$ tienten an. Daher ist eine telefonische Terminvereinbarung unbedingt notwendig, um beispielsweise Frühgeborene schützen zu können.

Ist die Anamnese bei Kindern oder Jugendlichen mit Durchfall wichtig? Die Anamnese kann wesentliche Hinweise darauf geben, ob ausreichend Flüssigkeit zugeführt wird und auch darüber wie der Krankheitsverlauf sich bisher entwickelt hat.

Wie gehen Sie diagnostisch bei Durchfall vor?

In aller Regel handelt es sich um eine klinische Diagnose die mit der Anamnese und einer körperlichen Untersuchung gestellt werden kann. Wesentlich ist die Einschätzung des Flüssigkeitsverlustes der bei kleinen Kindern mitunter beträchtlich sein kann.
Was steht aus Ihrer Sicht bei der Behandlung von Durchfall an erster Stelle?

Der Ersatz verlorener Flüssigkeiten. Langsamer Kostaufbau ist von Bedeutung, ebenso die Fiebersenkung.

Was raten Sie den Eltern für die Behandlung zu Hause?

Vor allem ist die ausreichende Hygiene von großer Wichtigkeit, weil die Erkrankung sonst unter den anderen Familienmitgliedern zirkulieren wird und auch darüber hinaus an andere Personen weitergegeben werden kann. Eine ausreichende Trinkmenge ist zu beachten sowie das Vermeiden von belastenden, etwa sehr süßen oder sehr fetten Speisen

Wie lange darf ein Durchfall bei Kindern/Jugendlichen dauern?

Meist dauert die Erkrankung etwa eine Woche. Bis der Stuhlfleck normalisiert ist, kann es jedoch noch etwas länger dauern.

Vielen Dank für das interessante Gespräch!

Hinweis des Verlags. Der Verlag bleibt in Hinblick auf geografische Zuordnungen und Gebietsbezeichnungen in veröffentlichten Karten und Institutsadressen neutral.

Paediatr. Paedolog. 2020 · 55:112-113 https://doi.org/10.1007/s00608020-00800-6

(c) Springer-Verlag GmbH Austria, ein Teil von Springer Nature 2020 
Hier steht eine Anzeige.

Springer 